\title{
Erratum to: Fas and FasL Expression in the Spinal Cord Following Cord Hemisection in the Monkey
}

\author{
Liu Jia $\cdot$ Zou Yu $\cdot$ Li Hui $\cdot$ Guan Yu-Guang • \\ Zhou Xin-Fu $\cdot$ You Chao $\cdot$ Xiyang Yanbin $\cdot$ Zhan Xi $\cdot$ \\ Wang Jun $\cdot$ Heng Xin-Hua $\cdot$ Wang Ting-Hua
}

Published online: 3 June 2011

(C) Springer Science+Business Media, LLC 2011

Erratum to: Neurochem Res (2011) 36:419-425

DOI 10.1007/s11064-010-0357-2

The co-author's name 'Hen Xin-Hua' was published incorrectly in the original publication of the article. The author's name should read as 'Heng Xin-Hua'

The online version of the original article can be found under doi:10.1007/s11064-010-0357-2.

L. Jia · W. Ting-Hua $(\bowtie)$

Institute of Neuronlogic Disease, West China Hospital, Sichuan

University, 610041 Chengdu, China

e-mail: tinghua_neuron@263.net

Z. Yu · L. Hui · G. Yu-Guang $\cdot$ Z. Xin-Fu $\cdot$ X. Yanbin .

W. Ting-Hua

Institute of Neuroscience, Kunming Medical College,

650031 Kunming, China

\section{Z. Xin-Fu}

Department of Human Physiology and Centre for Neuroscience, Flinders University, GPO Box 2100, Adelaide, SA 5001,

Australia

Y. Chao

Department of Neurosurgery, West China Hospital, Sichuan University, 610041 Chengdu, China

Z. Xi · W. Jun · H. Xin-Hua

Department of Anesthesiology, Kunming Medical College,

650031 Kunming, China 